\title{
Electron-photon deep inelastic scattering at small $x$ in holographic QCD
}

\author{
Akira Watanabe* \\ Institute of High Energy Physics and Theoretical Physics Center for Science Facilities, Chinese \\ Academy of Sciences, Beijing 100049, People's Republic of China \\ and \\ University of Chinese Academy of Sciences, Beijing 100049, People's Republic of China \\ E-mail: akira@ihep.ac.cn
}

\section{Hsiang-nan Li}

Institute of Physics, Academia Sinica, Taipei 11529, Taiwan, Republic of China

E-mail: hnliephys.sinica.edu.tw

\begin{abstract}
We study the electron-photon deep inelastic scattering at small Bjorken variable $x$ in the framework of holographic QCD, employing the Pomeron exchange to describe the involved strong interaction. With the Brower-Polchinski-Strassler-Tan Pomeron exchange kernel and appropriate wave functions for the incident and target particles, which are defined in the five-dimensional AdS space, we obtain the photon structure functions. It is shown that our predictions agree with the experimental data measured at LEP and with those derived from a known parameterization of the photon parton distribution functions.
\end{abstract}

XXVI International Workshop on Deep-Inelastic Scattering and Related Subjects (DIS2018)

16-20 April 2018

Kobe, Japan

\footnotetext{
${ }^{*}$ Speaker.
} 


\section{Introduction}

Although a photon is a fundamental particle, it is possible to investigate its internal structure since a photon may fluctuate into quark-antiquark pairs in high energy scattering processes. This investigation can be realized with the electron-photon deep inelastic scattering (DIS), and the quark-gluon structure of the photon can be explored through the structure functions. Comprehensive reviews for photon structure functions were given in Refs. [1, 2]. Historically, the electronphoton DIS is a cleaner process compared to other high energy ones involving hadrons as the initial states, and the corresponding experimental data have provided theorists valuable opportunities to test the perturbative techniques of QCD in the region of a large Bjorken variable $x$. The situation is completely different in the small $x$ region, where a photon can no longer be regarded as a point particle: its nature as a vector meson becomes dominant, which should be described by effective models, such as the vector meson dominance model.

In this work, we will study the photon structure functions at small $x$ in the framework of holographic QCD, which is an effective approach of QCD based on the AdS/CFT correspondence. It is known that the Pomeron, corresponding to the closed string sector in the string theory, and identified as a graviton in the higher dimensional curved space, gives a dominant contribution in the small $x$ region. There are various descriptions of the Pomeron in the literature on holographic QCD. Here we adopt the Pomeron exchange kernel proposed by Brower, Polchinski, Strassler, and Tan (BPST) [3]. Experimental data of the electron-nucleon DIS indicate that the Pomeron nature transits, depending on the probe photon four-momentum squared $Q^{2}$, from the so-called soft to hard Pomeron. It has been demonstrated $[4,5,6]$ that the BPST kernel, as applied to DIS at small $x$, well reproduces this transition.

A key issue is how to treat the target photon in the higher dimensional background, for which we take the following two approaches. The wave function of the five-dimensional $\mathrm{U}(1)$ vector field [7], which couples to a lepton at the UV boundary, is applicable to the probe virtual photon. In our first approach, we utilize this $\mathrm{U}(1)$ vector field to describe the target photon with very tiny four-momentum squared [8]. The second approach is based on the vector meson dominance model: we calculate the real photon structure functions by using the gravitational from factor of the $\rho$ meson constructed from a bottom-up AdS/QCD model [9]. The three adjustable parameters of the model all reside in the BPST kernel. Two of them, controlling the energy dependence of a cross section and the strength of the confinement effect, have been determined in the previous study on the nucleon DIS with the input of the proton structure function $F_{2}$ measured by HERA [10]. Therefore, the results presented here can be regarded as our predictions, although the overall factor needs to be tuned to fit the experimental data of the OPAL collaboration at LEP [11].

We show that our predictions in the two approaches are consistent with each other, and agree with the data and those derived from a known parameterization of the photon parton distribution functions (PDFs) [12]. The above observation implies that the vector meson dominance is realized in the present model setup, and that our framework is appropriate for the study of small $x$ physics. Besides, our predictions can be tested at future linear colliders, such as the planned International Linear Collider. 


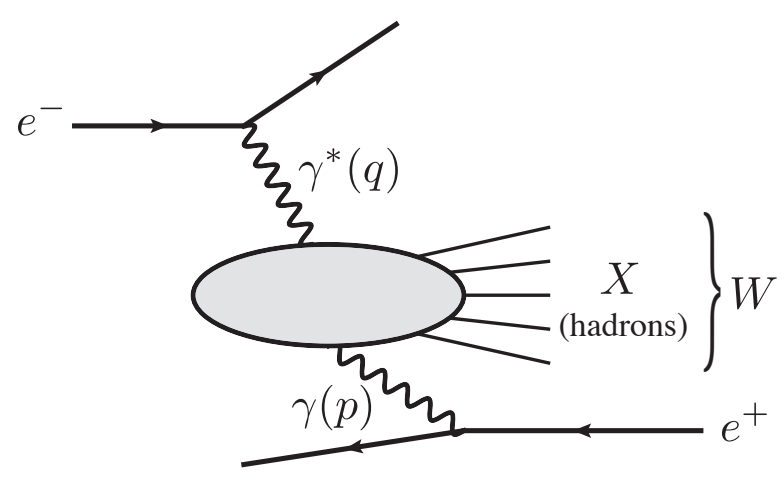

Figure 1: Electron-photon deep inelastic scattering.

\section{Model setup}

We analyze the electron-photon DIS schematically shown in Fig. 1, where $q$ and $p$ denote the four-momenta of the probe and target photons, respectively, and $W$ is the invariant mass of the hadronic final state $X$. For the real photon structure in the unpolarized case, we have the differential cross section

$$
\frac{d^{2} \sigma_{e \gamma \rightarrow e X}}{d x d Q^{2}}=\frac{2 \pi \alpha^{2}}{x Q^{4}}\left[\left\{1+(1-y)^{2}\right\} F_{2}^{\gamma}\left(x, Q^{2}\right)-y^{2} F_{L}^{\gamma}\left(x, Q^{2}\right)\right],
$$

in which $\alpha$ and $y$ are the fine structure constant and the inelasticity, respectively. The Bjorken scaling variable $x$ is defined, in terms of $Q^{2}=-q^{2}$ and $P^{2}=-p^{2}$, by

$$
x=\frac{Q^{2}}{Q^{2}+W^{2}+P^{2}} .
$$

Since we concentrate on the kinematic region with $W^{2} \gg Q^{2} \gg P^{2}$, the above definition can eventually be approximated by $x \approx Q^{2} / W^{2}$.

The two structure functions are expressed, with the BPST Pomeron exchange kernel $\chi$ in our model setup, as

$$
F_{i}^{\gamma}\left(x, Q^{2}\right)=\frac{\alpha g_{0}^{2} \rho^{3 / 2} Q^{2}}{32 \pi^{5 / 2}} \int d z d z^{\prime} P_{13}^{(i)}\left(z, Q^{2}\right) P_{24}\left(z^{\prime}, P^{2}\right)\left(z z^{\prime}\right) \operatorname{Im}\left[\chi\left(W^{2}, z, z^{\prime}\right)\right], \quad i=2, L,
$$

where the overall factor $g_{0}^{2}$ controls the magnitude of the structure functions, and $\rho$ governs their energy dependence. We employ the modified kernel for Eq. (2.3),

$$
\begin{aligned}
& \operatorname{Im}\left[\chi_{\text {mod }}\left(W^{2}, z, z^{\prime}\right)\right] \equiv \operatorname{Im}\left[\chi_{c}\left(W^{2}, z, z^{\prime}\right)\right]+\mathscr{F}\left(z, z^{\prime}, \tau\right) \operatorname{Im}\left[\chi_{c}\left(W^{2}, z, z_{0}^{2} / z^{\prime}\right)\right], \\
& \mathscr{F}\left(z, z^{\prime}, \tau\right)=1-2 \sqrt{\rho \pi \tau} e^{\eta^{2}} \operatorname{erfc}(\eta), \\
& \eta=\left(-\log \frac{z z^{\prime}}{z_{0}^{2}}+\rho \tau\right) / \sqrt{\rho \tau} .
\end{aligned}
$$

The first term on the right-hand side of Eq. (2.4) is the kernel from the conformal field theory,

$$
\operatorname{Im}\left[\chi_{c}\left(W^{2}, z, z^{\prime}\right)\right]=e^{(1-\rho) \tau} e^{-\left[\left(\log ^{2} z / z^{\prime}\right) / \rho \tau\right]} / \sqrt{\tau}
$$


with $\tau=\log \left(\rho z z^{\prime} W^{2} / 2\right)$. The second term on the right-hand side of Eq. (2.4) mimics the strength of the confinement effect adjusted by the parameter $z_{0}$. It has been found $[5,6]$ that one can have results from Eq. (2.4) in better agreement with the data of the nucleon structure functions, compared to those from Eq. (2.7).

The functions $P_{13}$ and $P_{24}$ in Eq. (2.3) represent the density distributions of the colliding particles in the five-dimensional AdS space. $P_{13}$ for the probe photon are set to the wave functions of the five-dimensional $\mathrm{U}(1)$ vector field,

$$
\begin{aligned}
& P_{13}^{(2)}\left(z, Q^{2}\right)=Q^{2} z\left\{K_{0}^{2}(Q z)+K_{1}^{2}(Q z)\right\} \\
& P_{13}^{(L)}\left(z, Q^{2}\right)=Q^{2} z K_{0}^{2}(Q z) .
\end{aligned}
$$

As mentioned in the Introduction, we take the same function as Eq. (2.8) for the target photon wave functions $P_{24}$ with tiny four-momentum squared [8] in the first approach. As to the vector meson dominance model in the second approach, we consider the photon- $\rho$ meson scattering with $P_{24}$ being described by the $\rho$ meson gravitational form factor in Ref. [9].

\section{Numerical results}

We present in Fig. 2 the behavior of the resulting photon structure function $F_{2}$ at small $x$. The solid and dashed curves represent our predictions for the photon-photon and photon- $\rho$ meson scattering, respectively, which agree with the LEP data [11] within the errors, and with those derived from the GRS PDF set at next-to-leading-order accuracy [12]. The agreement implies that the BPST Pomeron exchange kernel works well to describe the photon structure in the considered nonperturbative kinematic region, and that the vector meson dominance is realized in the present model setup.

Some ratios of the structure functions are exhibited in Fig. 3 for a more detailed comparison between the two approaches. The dependence of the ratio $R_{\gamma / N}=F_{2}^{\gamma}\left(x, Q^{2}\right) /\left[\alpha F_{2}^{N}\left(x, Q^{2}\right)\right]$ on the Bjorken variable $x$ is plotted in the panel (a), where the results for the nucleon structure function $F_{2}^{N}\left(x, Q^{2}\right)$ are taken from Ref. [6]. The ratios for the photon-photon scattering reveal opposite $x$ dependencies between the $Q^{2}=1$ and $10 \mathrm{GeV}^{2}$ cases, such that their difference increases with $x$. Both the $x$ and $Q^{2}$ dependencies of the ratios for the photon- $\rho$ meson scattering are quite weak. These features may be attributed to the fact that the $\rho$ meson is a normalizable mode, while the photon, described by Eq. (2.8), is not. In the panel (b), we display the longitudinal-to-transverse ratio of the structure functions $R_{L / T}=F_{L}\left(x, Q^{2}\right) / F_{T}\left(x, Q^{2}\right)$, where $F_{T}=F_{2}-F_{L}$, for $Q^{2}=1$ and $10 \mathrm{GeV}^{2}$ in the two approaches. It is seen that the four ratios slightly increase with $x$ and with $Q^{2}$. These behaviors agree qualitatively with those observed in the previous analysis of the nucleon DIS [6].

\section{Summary}

In this work, we have investigated the electron-photon DIS at small $x$ in holographic QCD. In addition to the photon-photon scattering, we have also taken into account the photon- $\rho$ meson scattering based on the vector meson dominance model. The structure functions obtained in the 


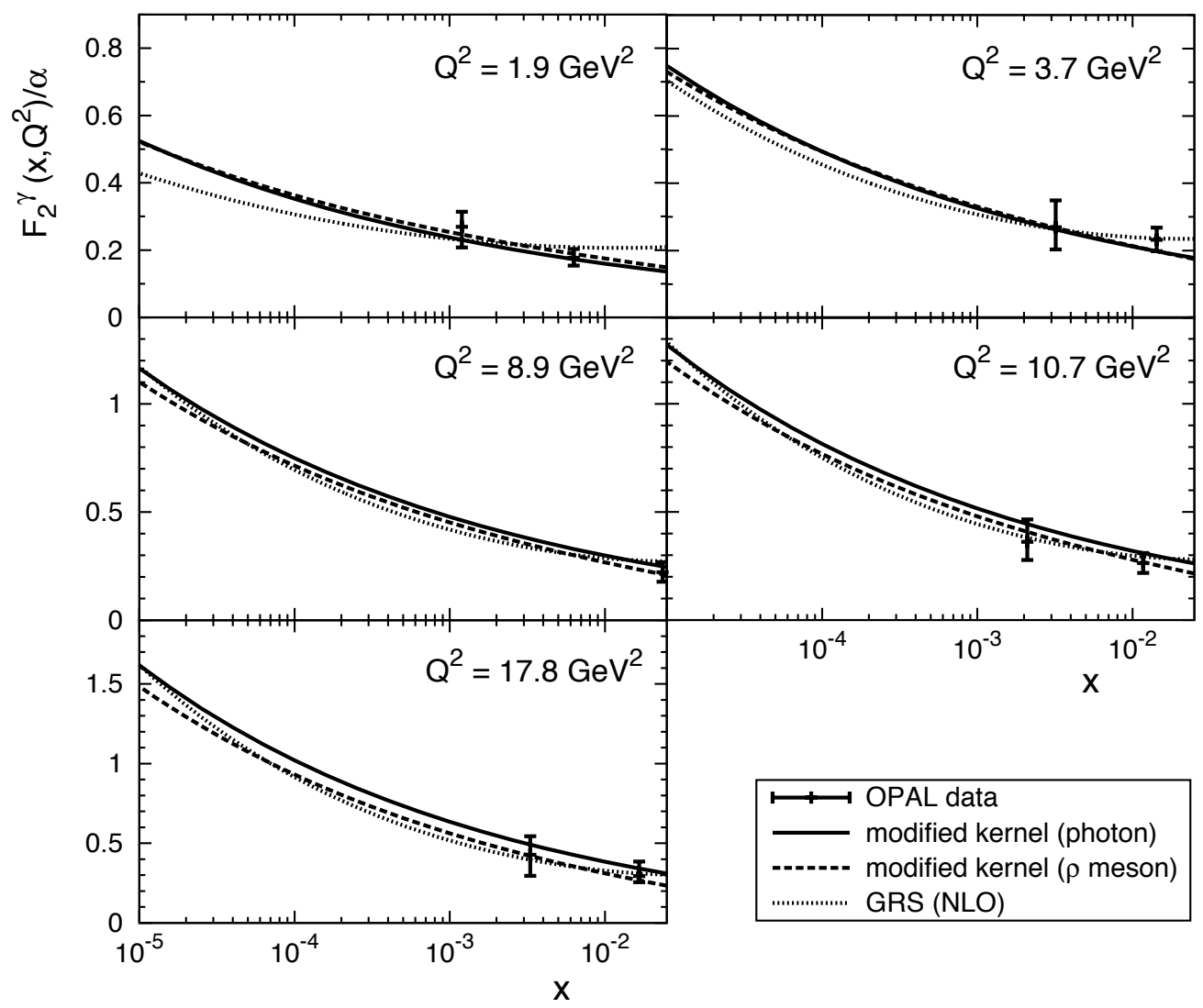

Figure 2: $F_{2}^{\gamma}\left(x, Q^{2}\right)$ as a function of the Bjorken variable $x$ for various $Q^{2}$. In each panel, experimental data measured by the OPAL collaboration at LEP [11] are displayed with error bars, the solid and dashed curves are from our calculations, and the dotted curves are from the PDF parameterization [12].

two approaches are consistent with each other, and with the LEP data and those from the PDF parameterization of the real photon in the considered kinematic region. The density distribution of the quasi-real photon, described by Eq. (2.8) with a tiny four-momentum squared, has a substantial component from the UV (small $z$ ) to IR (large $z$ ) region, while that of the $\rho$ meson has most of its component in the IR region. Hence, our findings indicate a nontrivial realization of the vector meson dominance in our model setup. The fact that the present holographic formalism works well in both the investigations of the photon and nucleon structures strongly supports its further applications to other high energy scattering processes, to which the conventional perturbation technique does not apply.

\section{References}

[1] R. Nisius, The Photon structure from deep inelastic electron photon scattering, Phys. Rept. 332 (2000) 165 [hep-ex/9912049].

[2] M. Krawczyk, A. Zembrzuski and M. Staszel, Survey of present data on photon structure functions and resolved photon processes, Phys. Rept. 345 (2001) 265 [hep-ph/ 0011083$].$ 
(a)

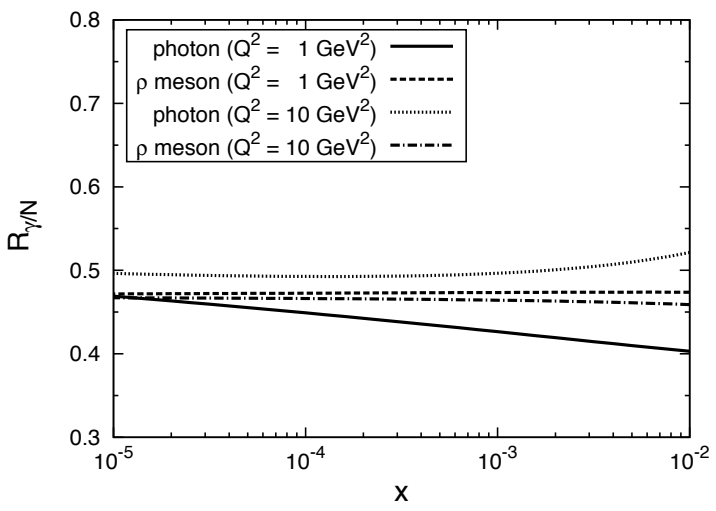

(b)

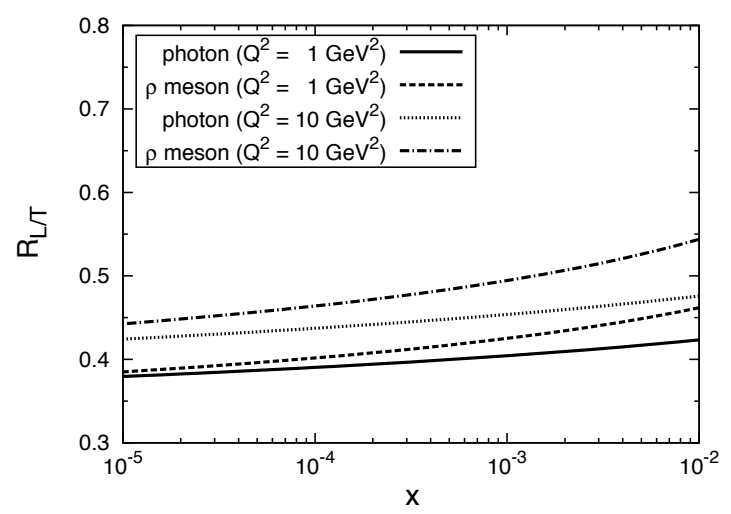

Figure 3: Ratios (a) $R_{\gamma / N}=F_{2}^{\gamma}\left(x, Q^{2}\right) /\left[\alpha F_{2}^{N}\left(x, Q^{2}\right)\right]$ and (b) $R_{L / T}=F_{L}\left(x, Q^{2}\right) / F_{T}\left(x, Q^{2}\right)$ as functions of the Bjorken variable $x$ for $Q^{2}=1$ and $10 \mathrm{GeV}^{2}$. The nucleon results $F_{2}^{N}\left(x, Q^{2}\right)$ are taken from Ref. [6], and common to all the curves in the panel (a).

[3] R. C. Brower, J. Polchinski, M. J. Strassler and C.-I. Tan, The Pomeron and gauge/string duality, JHEP 0712 (2007) 005 [hep-th/ 0603115$].$

[4] R. C. Brower, M. Djuric, I. Sarcevic and C.-I. Tan, String-Gauge Dual Description of Deep Inelastic Scattering at Small-x, JHEP 1011 (2010) 051 [1007.2259].

[5] A. Watanabe and K. Suzuki, Transition from soft- to hard-Pomeron in the structure functions of hadrons at small-x from holography, Phys.Rev. D86 (2012) 035011 [1206. 0910].

[6] A. Watanabe and K. Suzuki, Nucleon structure functions at small x via the Pomeron exchange in AdS space with a soft infrared wall, Phys. Rev. D89 (2014) 115015 [1312 . 7114 ].

[7] J. Polchinski and M. J. Strassler, Deep inelastic scattering and gauge / string duality, JHEP 0305 (2003) 012 [hep-th/0209211].

[8] A. Watanabe and H.-n. Li, Photon structure functions at small x in holographic QCD, Phys. Lett. B751 (2015) 321 [1502.03894].

[9] Z. Abidin and C. E. Carlson, Gravitational form factors of vector mesons in an AdS/QCD model, Phys. Rev. D77 (2008) 095007 [0 801 . 3839].

[10] F. Aaron et al., Combined Measurement and QCD Analysis of the Inclusive e+- $p$ Scattering Cross Sections at HERA, JHEP 1001 (2010) 109 [0911 . 0884].

[11] G. Abbiendi et al., Measurement of the low x behavior of the photon structure function F(2)gamma, Eur. Phys. J. C18 (2000) 15 [hep-ex/0007018].

[12] M. Gluck, E. Reya and I. Schienbein, Radiatively generated parton distributions of real and virtual photons, Phys. Rev. D60 (1999) 054019 [hep-ph/9903337]. 